REGARDS

SUR L'ECONOMIE ALLEMANDE

BULLETIN ECONOMIQUE DU CIRAC

\section{Regards sur l'économie allemande}

Bulletin économique du CIRAC

$100 \mid 2011$

Varia

\title{
30 ans de CIRAC, 20 ans de Regards sur l'économie allemande
}

par Alfred Grosser, Président du CIRAC

\section{Alfred Grosser}

\section{OpenEdition}

\section{Journals}

Édition électronique

URL : http://journals.openedition.org/rea/4215

DOI : $10.4000 /$ rea.4215

ISBN : 978-2-8218-0894-2

ISSN : 1965-0787

Éditeur

CIRAC

Édition imprimée

Date de publication : 17 mars 2011

Pagination : 1-8

ISSN : 1156-8992

\section{Référence électronique}

Alfred Grosser, " 30 ans de CIRAC, 20 ans de Regards sur l'économie allemande », Regards sur

l'économie allemande [En ligne], 100 | mars 2011, mis en ligne le 01 mars 2013, consulté le 22 septembre 2020. URL : http://journals.openedition.org/rea/4215 ; DOI : https://doi.org/10.4000/rea. 4215 


\section{0 ans de CIRAC, 20 ans de Regards sur l'économie allemande}

\section{par Alfred Grosser, Président du CIRAC}

Un chancelier et deux présidents : pour une fois, une belle continuité française. Helmut Schmidt avec Valéry Giscard d'Estaing, Helmut Schmidt avec François Mitterrand décident de la création d'un organisme français qui deviendra le Centre d'information et de recherche sur l'Allemagne contemporaine. Poussant loin un libéralisme devenu presque inimaginable dans la France d'aujourd'hui, ils confient la mise en route à une équipe fondatrice composée de personnalités différentes dans leurs convictions politiques, mais toutes connues pour leur action dans le domaine franco-allemand: Joseph Rovan, Pierre Bertaux, Jacques Bariéty, François G. Dreyfus, Alfred Grosser.

L'idée centrale est de créer une institution qui ressemble au Deutschfranzösisches Institut, organisme allemand créé à Ludwigsburg en 1948. Malheureusement, les trois hommes d'Etat ne sont pas aussi prévoyants que Charles de Gaulle et Konrad Adenauer en 1963/64. L'Office franco-allemand pour la Jeunesse bénéficie depuis sa création d'une ligne budgétaire, alors que le CIRAC, comme des centaines d'autres associations françaises, doit mendier chaque année le renouvellement de ses maigres subsides publics. Et, comme la France n'est pas l'Allemagne, la Wirtschaft, les dirigeants des entreprises privées, ne font pas preuve de la même clairvoyance ni du même engagement que les industriels du Wurtemberg dont l'argent a entièrement financé à ses débuts l'Institut de Ludwigsburg.

$\mathrm{Au}$ cours de la réunion constitutive du CIRAC, trois choix de personnes sont effectués. Pierre Bertaux sera le président, Alfred Grosser le président délégué, René Lasserre le secrétaire général. Le moins qu'on pouvait dire de Bertaux, c'est que, germaniste fils de germaniste, il n'avait pas mené une carrière universitaire tranquille. Avant-guerre, des cabinets ministériels, pendant la guerre, la Résistance, particulièrement active à Toulouse, après la guerre une préfecture régionale, la direction de la Sûreté nationale, l'entrée au parlement comme sénateur du Soudan. Puis le retour à l'Université comme germaniste d'un type particulier, parce que prioritairement intéressé par l'Allemagne la plus contemporaine. Il en résultera, en 1968, la création, au sein de l'Université de Paris (ultérieurement Paris III Sorbonne nouvelle), de l'Institut d'Allemand d'Asnières qu'il dirigera jusqu'à sa mort en 1986.

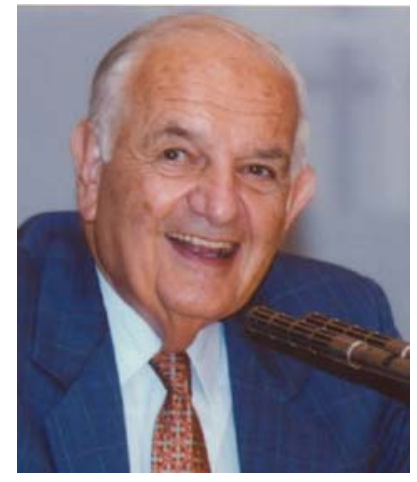

Alfred Grosser, professeur émérite, Sciences Po, Paris. 
Pierre Bertaux a lancé une formule qu'il savait lui-même outrancière, à savoir que les étudiants devaient connaître Karl Schiller, ministre de l'Economie à Bonn de 1966 à 1972 et aussi des Finances en 1971/72, plutôt que Friedrich Schiller, le grand écrivain ami de Goethe. Or, en 2011, à peu près personne, en France et même en Allemagne, ne sait plus qui était Karl, alors que même les écoliers savent que L'Hymne à la Joie, devenu chant européen, a été composé par Beethoven sur un poème de Friedrich. Et quel amateur de Verdi ignore que l'opéra Don Carlos est fondé sur une tragédie du même Friedrich Schiller ? (Malheureusement peu savent que la fin ridicule de l'opéra - Charles Quint sortant de sa tombe pour y entraîner l'infant - est de Verdi, pas de Schiller qui arrête magnifiquement le drame par la remise de l'héritier du trône par le roi son père au Grand Inquisiteur). Bertaux lui-même avait de fortes raisons de ne pas négliger la culture des poètes et des musiciens. Il était un grand spécialise de Hölderlin et avait, dans son adolescence, lié amitié, à Sèvres, avec le jeune Yehudi Menuhin.

Aux obsèques de Pierre Bertaux, dans l'émouvante petite église parisienne Saint Julien le Pauvre, il y eut d'abord les discours convenus et lus d'universitaires, puis, soudain, surgit Menuhin. Il fit d'abord, sans texte ni notes, un bref et bel hommage au défunt, puis, accompagné de trois instrumentistes qu'il avait amenés, joua du Bach. Mais Menuhin, de son côté, n'était pas seulement un artiste. Ou, plutôt, il l'avait été jusqu'à un voyage en Afrique du Sud où il avait découvert l'apartheid et la souffrance des hommes. Il en est résulté un engagement moral et social qui le fit jouer pour les rescapés des camps d'apatrides, pour les survivants juifs, pour les Allemands dans le désarroi. Et créer, avec sa sœur, une école de musique dans un quartier déshérité de Londres.

De mon côté, germaniste devenu professeur de Science politique, j'avais été secrétaire général du comité français d'échanges avec l'Allemagne nouvelle - créé en 1948 en liaison immédiate avec Ludwigsburg. Je n'hésite pas devant l'auto-citation, parce que les deux textes suivants illustrent encore aujourd'hui "les deux conceptions possibles de l'action culturelle française à l'étranger ", comme je l'écrivais dans l'éditorial du numéro de juin 1949 de notre bulletin d'information Allemagne. "L'une est celle de l'Alliance française et de la plupart des instituts français en Europe et dans les Amériques. Il s'agit là d'apporter des informations, des réalisations d'ordre artistique et littéraire à une élite francophone peu nombreuse. (...) L'autre conception est celle des contacts en profondeur s'adressant aussi bien aux mouvements de jeunesse qu'aux écrivains et artistes, aux syndicats qu'aux universités. (...) Il ne s'agit donc pas, comme dans un pays stable, de faire de la 'propagande culturelle'. Il s'agit, dans l'état de fermentation où se trouve l'Allemagne, d'aider les éléments les plus neufs, les plus valables. (...) L'aide la plus efficace, ce sont les contacts et échanges faits sur un pied d'égalité qui l'apportent (...). Il faut espérer que la réforme imminente de notre système d'occupation tiendra compte de l'utilité, de la nécessité des rencontres et échanges".

La vraie réforme eut lieu avec la fin de l'occupation, dans la perspective des Accords de Paris - et elle ne se fit pas du tout dans le sens espéré, ce qui m’amena, dans le numéro de mars 1954, sous le titre "La politique culturelle française à un tournant ", à rédiger un éditorial assez agressif protestant contre la "normalisation" des relations culturelles franco-allemandes. Elle consistait non à faire profiter les autres pays de ce qui avait été l'originalité des actions franco-allemandes, mais de réduire la présence française à la traditionnelle activité de nos Instituts culturels. "... Tout autres sont les contacts qui existent avec l'Allemagne 
(...). Ils s'adressent aux groupes les plus divers - mouvements de jeunesse, syndicats, industriels, techniciens, hommes politiques, étudiants, journalistes, etc. Leur but n'est pas de faire une sorte de propagande culturelle française - nécessaire sans doute, mais de portée limitée-, mais de développer dans chaque pays la connaissance des réalités essentielles de l'autre... ". Mon texte me valut des critiques acerbes du ministère des Affaires étrangères, mais cela faisait déjà longtemps que l'Institut de Ludwigsburg se voyait faire la guerre, par l'Institut français de Stuttgart et depuis Paris, à cause de sa liberté d'action, de son indépendance, de sa possibilité de faire appel à des personnalités et des organismes français de son choix.

Il était clair, en 1982, que c'est dans ce sens que devait agir le CIRAC. Aussi avons-nous choisi comme secrétaire général un homme jeune (trente-six ans), ayant la double formation Asnières/Sciences Po et fort de ses travaux sur la société et l'économie allemandes. Nous ne pensions pas, je l'avoue, que René Lasserre ferait, parallèlement à son inlassable activité pour le CIRAC, une carrière universitaire aussi brillante. D'abord assistant puis maître de conférences à Asnières et chargé d'enseignement à l'IEP de Paris, il est devenu professeur de civilisation allemande à la jeune université de Cergy-Pontoise. Il y a si bien réussi, notamment en créant des formations débouchant sur des emplois dans les entreprises de la région et à l'international, que ses collègues l'ont porté à la vice-présidence, avant de lui confier, de 1999 à 2004, les pouvoirs de président de l'Université. Il n'était que justice que, de secrétaire général, il soit devenu directeur du CIRAC. Le président que je suis depuis la mort de Pierre Bertaux s'est simplement soucié de soutenir de son mieux l'action de René Lasserre, en coopération fréquente avec Ludwigsburg et avec la Fondation Bosch, prenant de multiples initiatives presque toujours abouties, fort du soutien de rares collaborateurs, en particulier, pour les médias, puis pour l'économie, d'Isabelle Bourgeois.

Le tout avec un souci financier de plus en plus aigu. Contrairement au président que j'étais et que je suis, René Lasserre est un honorable money raiser. Mais il n'a jamais eu le goût du spectaculaire qui, seul, plaît aux politiques, et les félicitations qu'il recevait des milieux économiques n'ont que rarement correspondu à un soutien financier. Du côté des autorités, nous avons trop souvent entendu, comme tant d'autres associations : "Pour être subventionnés, ayez des ressources propres " "Ah, vous avez des ressources propres ? Alors vous n'avez pas besoin de subventions". Le comble a été atteint en 2009/2010. Je ne crains pas de narrer l'anecdote. Le ministère des Affaires étrangères nous avait annoncé que notre (petite) subvention serait supprimée. Le 9 novembre, je suis à l'Arc de Triomphe puis dans un salon du Quai d'Orsay où nous attendons le ministre avant de passer à table. Bernard Kouchner arrive, fonce vers moi, me tutoie, m'appelle par mon prénom, puis, à la fin du repas, m'exprime l'espoir d'une prochaine rencontre. Dès le lendemain, je lui envoie une lettre disant que les sentiments amicaux qu'il me portait devraient avoir pour effet le rétablissement de la subvention du CIRAC dont j'étais le président. Il y eut une réponse. Elle disait, en toute simplicité, que nous travaillions si bien que nous n'avions pas besoin de subventions!

En difficulté financière presque constante, malgré le désintéressement de son directeur, malgré le nombre sans cesse diminué de ses collaborateurs, le CIRAC, en trente années d'existence, a à son actif un nombre remarquable de réalisations, qu'il s'agisse de publications ou de rapports d'études. L'estime et la confiance qui lui sont portées ont permis nombre d'enquêtes sur les universités et sur divers aspects de la société 
industrielle dans les deux pays. La même estime et la même confiance ont porté René Lasserre à la présidence du conseil d'administration du CIERA, le Centre interdisciplinaire d'études et de recherches sur l'Allemagne qui regroupe nombre d'universités et de centres de recherche français, dont Paris I, Paris IV, Lyon III, l'Université de Cergy-Pontoise, l'EHESS, les Ecoles normales supérieures, la Maison des Sciences de l'Homme.

Tout le monde nous dit qu'il faut absolument continuer. Mais presse, radios, télévisions, même s'ils font souvent appel à l'expertise de René Lasserre ou d'Isabelle Bourgeois, présentent rarement l'action du CIRAC. Ses publications sont utilisées, mais les informations et analyses ne sont pas répercutées en fonction de leur origine. On se tromperait en croyant que je me contente de doléances. Je suis simplement conscient de ce que le CIRAC, par son potentiel d'expertise et le travail de fond que représente depuis vingt ans sa revue Regards sur l'économie allemande, pourrait apporter en supplément de ce qu'il apporte déjà pour la connaissance de tous les aspects de l'Allemagne actuelle. Cela à un moment où l'Allemagne est de nouveau au centre des préoccupations françaises, malheureusement sur arrière-plan de bien des ignorances et de mythes sans cesse renaissants.

\author{
Le CIRAC a été fondé le $1^{\mathrm{er}}$ mars 1982 à Paris, \\ à la suite d'une déclaration commune \\ du Président de la République française (Valéry Giscard d'Estaing) \\ et du Chancelier fédéral (Helmut Schmidt) \\ lors du Sommet franco-allemand du 6 février 1981. \\ Sa création a été officiellement annoncée \\ par le Président de la République française (François Mitterrand) \\ le 20 janvier 1983, lors de son discours devant le Bundestag.
}

Extrait de la Déclaration franco-allemande en matière culturelle du 6 février 1981 :

\title{
"Centre d'information et de recherche sur l'Allemagne contemporaine.
}

Un centre d'information et de recherche sur l'Allemagne contemporaine sera créé. Ce centre, de niveau universitaire, aura pour mission de susciter, coordonner et développer les études sur l'Allemagne actuelle. Il ne se substitue à aucun des organismes existants, mais aura pour tâche d'assurer entre eux la coordination nécessaire. Il fournira en même temps à tous les usagers intéressés, y compris les collectivités locales, les entreprises, etc., une information sur les réalités scientifiques, techniques, culturelles, économiques et politiques allemandes. Il facilitera les échanges universitaires entre les deux pays et offrira un support aux enseignements de langues vivantes appliquées, dispensés dans les universités".

Source : Présidence de la République - Service de presse. Texte complet publié in Les relations franco-allemandes depuis 1963. Documents rassemblés et présentés par Pierre Jardin et Adolf Kimmel, La Documentation française, Paris, 2001 (pp. 242-244).

La version allemande est publiée in Presse und Informationsamt der Bundesregierung, Bulletin, Nr. 12, Bonn, 11 février 1981, pp. 101-103. 


\section{Historique du CIRAC}

6 février 1981 : Annonce de la création d'un CIRAC dans la Déclaration francoallemande en matière culturelle lors du Sommet franco-allemand

$1^{\text {er }}$ mars 1982 : Création du CIRAC à Paris, implanté initialement à la Maison des Sciences de l'Homme

Janvier 1983 : Inauguration des bureaux du CIRAC au 9, rue de Téhéran, Paris $8^{\mathrm{e}}$

Création de la bibliothèque du CIRAC (son centre de documentation sur l'économie et la société allemandes compte aujourd'hui près de 10000 ouvrages et quelque 200 périodiques, la plupart en langue allemande).

Lancement des collections d'ouvrages :

- Les Cahiers du CIRAC, qui donneront, peu après, naissance à la collection d'ouvrages Travaux et Documents du CIRAC

-Deutsch-französische Studien zur Industriegesellschaft aux Editions Campus (Francfort-Main/New-York) ; Directeurs de la coll. : Leo Kißler et René Lasserre.

\section{Lancement des périodiques :}

- CIRAC-Forum. Bulletin pour la coopération franco-allemande dans les sciences humaines et sociales. Créée en 1988, cette revue est aujourd'hui publiée en ligne (4 numéros par an)

- CIRAC-Media. Media et Communication en RFA ; bulletin trimestriel (1990-1993) - Regards sur l'économie allemande - Bulletin économique du CIRAC. Créée en mars 1991, cette revue (5 numéros par an) est également consultable en ligne depuis 2007 sur www.revues.org, et depuis 2010 sur www.cairn.info.

Organisation de colloques, en coopération avec le Deutsch-Französisches Institut de Ludwigsburg (DFI) et d'autres partenaires, notamment :

-1983, Berlin (Reichstag) : L'Allemagne en Europe : la problématique de l'identité nationale (CIRAC, DFI)

-1984 : Les stratégies de la RFA et de la France au sein de la Communauté européenne (CIRAC, Groupe « Europe » du Commissariat général du Plan, Institut für Europäische Politik)

-1984 : La contribution de la France et de la République fédérale d'Allemagne à l'unification européenne (CIRAC, DFI, DGAP)

-1985, Berlin (Reichstag) : Changements socio-culturels et légitimité politique en RFA et en France (CIRAC, DFI, Bundespresseamt, Robert-Bosch-Stiftung)

-1986, Versailles : Forum Culturel franco-allemand, Volet « Langue, éducation et recherche en sciences humaines et sociales »

-1987: L'enjeu européen: sécurité, technologie, politique spatiale (CIRAC, DGAP, DFI, IFRI, Senat de Hambourg)

-1988 : Structures, niveaux et programmes de formation des ingénieurs et techniciens en Europe (CIRAC, Bureau GE-TH, avec le soutien de la Commission des Communautés européennes)

-1988, à l'occasion du $40^{\mathrm{e}}$ anniversaire de l'Institut Franco-Allemand de Ludwigsburg (DFI) : Une Alliance dans l'Alliance ? Les relations franco-allemandes et l'Europe (CIRAC, DFI)

-Mai 1990, Berlin (Reichstag) : L'Allemagne et la France dans la nouvelle Europe (CIRAC, DFI, DGAP).

Organisation de séminaires et groupes de travail sur les politiques industrielles, technologiques, le partenariat social, le management, la $\mathrm{GRH}$, l'automatisation et les process de production industrielle, la formation professionnelle, les politiques d'éducation et de formation, l'innovation, les médias et les TIC, l'économie du savoir, les dérégulations, les mutations sociales, la politique européenne...

- Par exemple, en 1987: Groupe de travail Pour une coordination franco-allemande des politiques européennes (CIRAC, DFI, Institut für Europapolitik, en partenariat avec : Robert-Bosch-Stiftung, DGAP, BIPE, FES, DGB, Laboratoire de Sociologie du Travail du CNAM, CNRS, Fernuni Hagen, SJTI, CSA, Thüringer Landesmedienanstalt, Hans-Böckler Stiftung, IFRI). 
Organisation de voyages d'études en Allemagne pour les entreprises françaises, en partenariat avec Entreprise \& Personnel, Instituts Fraunhofer IPA et ISI, EdF, Centre National des Caisses d'Epargne, CEGOS, Centre Européen des Entrepreneurs publics...

- Par exemple, en 1991 : Voyage d'étude de l'Ecole des Cadres du Groupe Elf Aquitaine sur L'Allemagne au lendemain de l'unification.

Organisation de séminaires pour journalistes français et allemands en coopération avec le DFI (notamment entretiens avec le Chancelier Helmut Kohl et le ministre fédéral des Affaires étrangères Hans-Dietrich Genscher sur la responsabilité internationale de l'Allemagne après l'Unité ; avec les responsables de la Bundesbank, des grandes banques privées allemandes et du syndicat IG Metall sur l'avenir de la coopération économique européenne depuis les Traités de Maastricht).

1994-2001 : Le CIRAC s'installe à Levallois-Perret.

Juillet 2001 : Le CIRAC s'installe auprès de I'Université de Cergy-Pontoise, dont René Lasserre est alors vice-président, puis président (1999-2004).

Organisation de projets d'études :

-2000-2002: Modernisation et innovation dans les universités en France et en Allemagne, en partenariat avec : Robert-Bosch-Stiftung, Ministère de l'Education nationale, Caisse des Dépôts et Consignations, et en coopération avec la CPU

-2002-2004 : Fonctions et missions des chefs d'établissements scolaires de l'enseignement secondaire en France et en Allemagne ». En coopération avec l'ESEN, le Land de Rhénanie-Palatinat et la Région Bourgogne, et avec le soutien de la Asko-Europa-Stiftung et de l'OFAJ.

Organisation du symposium Dialogue franco-allemand sur les médias (Berlin, 2006), en coopération avec la Thüringer Landesmedienanstalt et avec le soutien de la Landesanstalt für Medien de Rhénanie du Nord-Westphalie

2001 : Constitution du GIP Centre Interdisciplinaire d'Etudes et de Recherche sur l'Allemagne (CIERA), dont le CIRAC est membre fondateur.

Les autres membres fondateurs en sont le CNRS, l'EHESS, L'Ecole normale supérieure de Lettres et Sciences humaines (Lyon), l'Ecole normale Supérieure (Ulm), la Fondation Maison des Sciences de l'Homme, l'Institut d'Etudes politiques de Grenoble, l'Université de Cergy-Pontoise, l'Université Lumière Lyon II, I'Université ParisSorbonne Paris IV et I'Université de Panthéon-Sorbonne Paris I.

René Lasserre est, depuis la fondation, président du Conseil d'Administration du GIP CIERA.

Organisation de débats et journées d'études :

-2002 : Les modèles français et allemands à l'épreuve de la compétitivité européenne

-2003 : Retraites, santé, chômage: Quelles réformes pour l'avenir de la protection sociale en Europe?

-2004 : Le système bancaire allemand face à la mondialisation : vers la fin d'un modèle?

Organisation de programmes de formation-recherche sous l'égide et avec le soutien du CIERA, en coopération avec d'autres partenaires :

-2004-2006 : La modernisation du management public en Allemagne (avec le soutien de la Fondation Hans-Böckler)

-2006-2008: Le système allemand d'innovation: organisation et dynamiques nouvelles. Eléments pour une comparaison France-Allemagne (avec le soutien de l'Université de Cergy-Pontoise, laboratoire CICC)

-2008-2010 : Relations professionnelles et régulation sociale dans les services d'intérêt général. Comparaison France-Allemagne (avec le soutien de l'Université de Cergy-Pontoise, laboratoire $\mathrm{CICC}$ )

-2010-2012 : Les politiques d'aide au retour à l'emploi des chômeurs en situation d'exclusion. Comparaison France-Allemagne.

En savoir plus : www.cirac.u-cergy.fr 


\title{
20 ans de Regards sur l'Allemagne unie
}

\author{
Sélection de dossiers et articles parus depuis mars 1991 \\ dans la revue Regards sur l'économie allemande
}

Rémi LaLlement, «Perspectives de l'économie est-allemande: Prévisions à long terme et handicaps initiaux - Potentiel productif de l'ex-RDA : l'ampleur du renouvellement nécessaire »

Rémi LALLEMENT, « Unification allemande : de nouveaux défis pour la politique économique - Emploi, revenus et compétitivité : la quadrature du cercle - Budgets publics et politique monétaire : une contradiction croissante »

Rudolf SCHARPING, "La Rhénanie-Palatinat: de la périphérie de l'Allemagne au centre de l'Europe »

Rémi LALLEMENT, « La communauté européenne entre approfondissement et élargissement : le débat vu d'Allemagne »

Kajo SCHOMMER, « L'éveil de la Saxe »

$\mathbf{N}^{\circ} \mathbf{1}(\operatorname{mars} 1991)$

$\mathbf{N}^{\circ} 2$ (juin 1991)

N 8 (oct. 1992)

N 9 (déc. 1992)

$\mathbf{N}^{\circ} 11$ (mai 1993)

Rémi LALLEMENT, « La compétitivité de l'Allemagne comme espace économique - Le made in Germany en question : I - Diagnostic »

Rémi LALLEMENT, « La compétitivité de l'Allemagne comme espace économique - Le made in Germany en question : II - Thérapie »

Rémi LALLEMENT, « La politique commerciale : une pomme de discorde entre l'Allemagne et la France ? »

Rémi Lallement, Kristin SPECK « Cohésion et aménagement du territoire en Allemagne »

Olivier FLOC'HIC, « La construction automobile en Allemagne. Compétitivité, technologie, réorganisation »

Olivier FLOC'HIC, « L'assurance dépendance en RFA : un financement qui suscite bien des débats ॥

Olivier FLOC'HIC, « Finances publiques et réforme de la fiscalité : au centre du débat sur la croissance »

Olivier FLOC'HIC, « Le commerce franco-allemand : 'noyau dur' du commerce extérieur des deux pays $»$

Olivier FLOC'HIC, «Le système bancaire allemand au cœur du débat sur la croissance »

Marie-Hélène PAUTRAT, « Le pacte pour l'emploi : pour une relance du dialogue social » Alain LATTARD, « Négociation collective : quel avenir pour la convention de branche ? »

Florence AUTRET, « L'innovation technologique en Allemagne : état des lieux et limites d'un système »

Markus GABEL, " De la banque-industrie aux industries bancaires - les mutations du système bancaire allemand »

Isabelle BOURGEOIS, « Dix ans après l'unification : l'économie des nouveaux Länder en voie de normalisation »

Brigitte LESTRADE, « L'emploi des femmes en Allemagne. Le temps partiel, une spécialité allemande?"

Ulrike MAYRHOFER, Christoph BARMEYER, « EADS et l'intégration des différences culturelles $»$

Henrik UTERWEDDE, « Feu le 'capitalisme rhénan' ? »

N 15 (mars 1994)

$\mathbf{N}^{\circ} 16$ (mai 1994)

N 17 (sept. 1994)

N 19 (déc. 1994)

$\mathbf{N}^{\circ} 20$ (mars 1995)

$\mathbf{N}^{\circ} 21$ (mai 1995)

$\mathbf{N}^{\circ} 26$ (mai 1996)

N²9 (déc. 1996)

N³2 (oct. 1997)

N 40 (mars 1999)

No 43 (oct. 1999)

N 44 (déc. 1999)

$\mathbf{N}^{\circ} \mathbf{4 5}$ (mars 2000)

$\mathbf{N}^{\circ} 46$ (mai 2000)

N 49 (déc. 2000)

N' 52/53 (oct. 2001)

$\mathbf{N}^{\circ} \mathbf{5 5}$ (mars 2002) 
N 56 (mai 2002) « Licenciement économique et pouvoir des juges en RFA. Un entretien avec le juge Meinhard ZUMFELDE ॥

№57 (juillet 2002) Isabelle BOURGEOIS, « Le 'miracle biotechnologique' allemand »

N 59 (déc. 2002) Cornelia APPEL, Michael SCHIPPERGES, « Les Allemands, l'euro et la mondialisation »

№ 60 (mars 2003) Christioph BARMEYER, « Management franco-allemand : gérer les 'incidents critiques' »

N 63 (oct. 2003) Patrick HASSENTEUFEL, « Agenda 2010, tournant pour l'assurance maladie »

N 65 (mars 2004) Florence AUTRET, « Une politique énergétique entre libéralisation et indépendance »

№67 (juillet 2004) Gabriel COLLETIS, « Mutation du 'modèle rhénan' et avenir du modèle européen »

N 70 (mars 2005) Stefanie WAHL, « Démographie et compétitivité »

№ 72 (juillet 2005) René LASSERRE, « La cogestion allemande à l'épreuve de la globalisation »

№ 75 (mars 2006) Werner ZETTELMEIER, « Allemagne : la transition formation/éducation/emploi »

№ 78 (oct. 2006) Wolfgang SCHRœEDER et Arijana NEUMANN, «Vieillissement démographique: entre déni et dramatisation »

№ 80 (mars 2007) Isabelle BOURGEOIS, « Opinions et valeurs : 60 ans de démoscopie allemande »

№ 82 (juillet 2007) Hans STARK, « Présidence allemande de l'UE : de la 'racine carrée' à la quadrature du cercle "

N 85 (mars 2008) Spécial dérégulation :

Isabelle BOURGEOIS, « Marché postal allemand : libéralisation sans concurrence »

Caroline GUIOT, « Deutsche Post AG : un géant mondial de la logistique »

N 86 (mai 2008) Isabelle BOURGEOIS, «Fret ferroviaire en RFA : une dynamique à optimiser »

№ 87 (juillet 2008) Dossier spécial Chine :

Solène HAZOUARD, « Des entreprises allemandes bien positionnées en Chine » Un entretien avec Monika STÄRK (OAV) : « Considérer la Chine avec le respect dû à un partenaire »

№ 88 (oct. 2008) Karl-Peter SCHACKMANN-FALLIS, « Les Sparkassen, facteur de stabilité pour l'économie allemande »

Stefanie WAHL, « Les vraies causes de la disparité des revenus en RFA »

№ 89 (déc. 2008) Joachim SCHILD, « La force de l'imprévisible : la présidence française de l'UE face à la crise financière. Un point de vue allemand »

№ 91 (mai 2009) Numéro Spécial 60 anniversaire de la RFA :

Alfred GROSSER, « Il était une fois un petit Etat provisoire... »

Henrik UTERWEDDE, « L'économie sociale de marché : la jeunesse d'un référentiel » Hans-Helmut KOTZ, « 60 ans de Loi fondamentale, 61 ans de Deutsche Mark » Thomas PETERSEN, « Une nouvelle identité allemande ?»

№ 92 (juillet 2009) Peter-Christian MüLLER-GRAFF, « L'arrêt de Karlsruhe sur le Traité de Lisbonne »

№ 94 (déc. 2009) René LASSERRE, « L’Allemagne à la recherche d'une politique de sortie de crise »

№ 95 (mars 2010) Ulrike REISACH, « Culture des affaires - GM et Opel : malentendus germano-américains » Géraldine DEMME, « Le secteur de la santé face au droit de la concurrence »

№ 96 (mai 2010) Markus Promberger, « 5 ans de loi Hartz IV : continuité et ruptures dans les politiques allemandes pour l'emploi et contre la pauvreté »

Winfried HEIDEMANN, « La formation continue en Allemagne »

№ 98-99 (oct.-déc. 2010) Dossier Spécial 20 ans d'Unité allemande :

Karl BRENKE, « 20 ans après l'Unité : regards sur l'économie dans l'est de l'Allemagne » Eugen SPITZNAGEL, « Le marché de l'emploi est-allemand 20 ans après l'Unité » Thomas PETERSEN, « Frères et sœurs dissemblables. Un bilan de l'Unité allemande » Uwe KAMMANN, «Compagnons de voyage. Les médias et l'Unité allemande » Isabelle BOURGEOIS, « Les médias dans l'Allemagne unie. De l'unification démocratique à la normalisation du marché ». 